SLAC-PUB-13408

BABAR-PROC-08/066

arXiv:0809.4145[hep-ex]

September, 2008

\title{
New Physics Searches at BABAR
}

\author{
F. Renga \\ Università di Roma "La Sapienza" and INFN Roma \\ (for the BABAR Collaboration)
}

\begin{abstract}
We will present the most recent results from the BABAR Collaboration concerning New Physics searches in rare $B$ and Lepton Flavour Violating (LFV) decays, including $b \rightarrow s$ transitions, purely leptonic $B$ decays and LFV $\tau$ decays.
\end{abstract}

Presented at the 16th International Conference On Supersymmetry And The Unification Of Fundamental Interactions (SUSY08) 06/16/2008 - 06/21/2008, Seoul, Korea 


\section{Introduction}

Rare $B$ and $\tau$ decays are a standard probe for New Physics (NP) searches and a $B$-Factory provides a unique environment to look for these processes. Many rare decays can be investigated and are sensitive to different NP scenarios.

At first, we can consider $B$ decays mediated by flavour-changing neutral currents (FCNC). They are characterized by low rates in the Standard Model (SM), due to the absence of FCNC at tree level. Hence, NP effects can be of the same order of magnitude of the SM contributions. The high number of $B \bar{B}$ pairs produced by a $B$-Factory often allows to approach or reach the experimental sensitivity needed to observe these decays, and strong constraints can be put on the NP contributions, by comparing the experimental results with the SM expectations.

We can also consider purely leptonic $B$ decays and LFV $\tau$ decays. The very low SM rate of these decays often make them unaccessible with the present experiments, unless NP effects enhance the rate up to the current experimental sensitivity. For some of these decays, just the observation by itself would provide an unambiguous evidence of NP.

In this work, we present the most recent NP searches in rare $B$ and $\tau$ decays, based on the data collected by the BABAR detector (1) at PEP-II, an asymmetric $e^{+} e^{-}$collider operating at a center of mass energy of $10.58 \mathrm{GeV}$, corresponding to the mass of the $\Upsilon(4 S)$ resonance.

\section{Electroweak Penguins}

The FCNC decays proceeding through electroweak penguin diagrams show a good sensitivity to supersymmetric models and other NP scenarios.

Among them, the $B \rightarrow X_{s} \gamma$ process has been deeply investigated both from the theoretical and experimental point of view in the last years. A SM estimate of the inclusive branching ratio (BR) is available at the NNLO (3):

$$
\mathcal{B}\left(B \rightarrow X_{s} \gamma\right)=(3.15 \pm 0.23) \times 10^{-4} .
$$

The CP asymmetry:

$$
A_{C P}=\frac{\Gamma\left(\bar{B} \rightarrow X_{s} \gamma\right)-\Gamma\left(B \rightarrow X_{\bar{s}} \gamma\right)}{\Gamma\left(\bar{B} \rightarrow X_{s} \gamma\right)+\Gamma\left(B \rightarrow X_{\bar{s}} \gamma\right)},
$$

is also interesting, since it is expected to be at the level of $0.5 \%$ in the SM but can be strongly enhanced by NP effects (4).

The $B A B A R$ Collaboration produced several results for the inclusive BR, adopting different techniques. The most stringent measurement, based on a sample of about $88.9 \times 10^{6} B \bar{B}$ pairs, has been done by means of a semi-inclusive approach (5) and gives:

$$
\mathcal{B}\left(B \rightarrow X_{s} \gamma\right)=\left(3.27 \pm 0.18_{-0.41}^{+0.55}\right) \times 10^{-4},
$$

where (here and in the following) the first error is statistical, the second is systematic. In this analysis, 38 exclusive final states are reconstructed (22 modes with 1 kaon and 1 to 4 pions, 10 modes with a kaon, an $\eta$ and up to 2 pions, 6 modes with 1 kaon and up to 1 pion). The signal yields are combined in order to extract the inclusive BR. The error is dominated by the systematic uncertainty coming from the modeling of the parton fragmentation and the contribution from unreconstructed modes. A similar technique has been used in order to measure the CP asymmetry, obtaining:

$$
A_{C P}=-0.011 \pm 0.030 \pm 0.014
$$




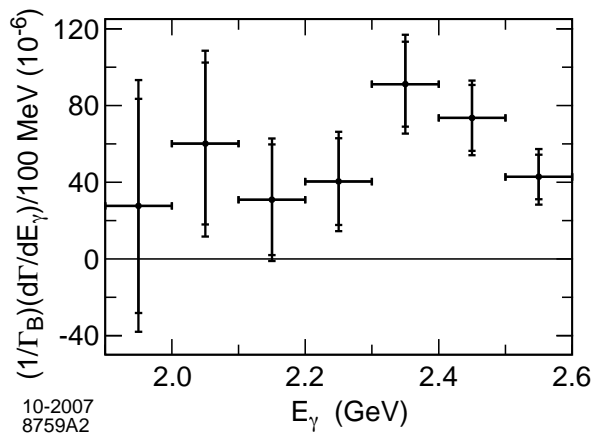

Figure 1: Partial $B \rightarrow X_{s} \gamma \mathrm{BR}$ in different regions of $E_{\gamma}$.

in a sample of about $383 \times 10^{6} B \bar{B}$ pairs (11).

Recently, the Collaboration produced a new result for the BR, based on a recoil technique (6). In this kind of analysis, one of the two $B\left(B_{t a g}\right)$ is reconstructed in a frequent decay mode, while the signal signature is searched for in the rest of the event (the recoil), composed by all tracks and neutral particles not associated to the $B_{t a g}$. This technique provides a pure sample of $B \bar{B}$ events and a clean environment to look for rare decays.

The $B \rightarrow X_{s} \gamma \mathrm{BR}$ is measured on the recoil of hadronic $B \rightarrow D X$ decays. More than 1000 exclusive $B_{t a g}$ decay modes are used. The kinematical consistency of the $B_{t a g}$ is checked with the two variables $m_{\mathrm{ES}}=\sqrt{E_{\text {beam }}^{* 2}-\left|\mathbf{p}_{B}^{*}\right|^{2}}$ and $\Delta E=E_{B}^{*}-E_{\text {beam }}^{*}$, where $E_{\text {beam }}^{*}$ is the beam energy and $E_{B}^{*}$ and $\mathbf{p}_{B}^{*}$ are the energy and the momentum of the $B_{\text {tag }}$ in the center of mass frame.

A photon with energy $E_{\gamma}>1.3 \mathrm{GeV}$ is required on the recoil. Finally, the signal yield is extracted by means of a Maximum Likelihood (ML) fit, based on the distribution of $m_{\mathrm{ES}}$. The BR measured from a sample of about $232 \times 10^{6}$ is:

$$
\mathcal{B}\left(B \rightarrow X_{s} \gamma\right)=(3.66 \pm 0.85 \pm 60) \times 10^{-4} .
$$

The fit is also performed in different bins of $E_{\gamma}$ in order to measure the photon energy spectrum. The results are shown in Fig. 1.

The FCNC $B \rightarrow X_{s} \ell^{+} \ell^{-}(\nu \bar{\nu})$ decays are also mediated by electroweak penguin diagrams, along with electroweak box diagrams. These processes are mainly sensitive to non-standard $Z$ couplings (7). Moreover, since the two neutrinos in the $B \rightarrow X_{s} \nu \bar{\nu}$ decays are not detected, the experimental search is also sensitive to some exotic sources of missing energy, like light dark matter (8) and Unparticles (9).

The BaBar Collaboration measured the inclusive $B \rightarrow X_{s} \ell^{+} \ell^{-}$BR by means of a semi-inclusive approach, by looking for exclusive modes with 1 kaon and up to 3 pions (10). The result is:

$$
\mathcal{B}\left(B \rightarrow X_{s} \ell^{+} \ell^{-}\right)=(5.6 \pm 1.5 \pm 1.3) \times 10^{-6},
$$

and is consistent with the SM expectations. Also in this case, large systematic uncertainties are present due to the fragmentation modeling.

Measurements concerning the exclusive $B \rightarrow K^{(*)} \ell^{+} \ell^{-}$decays have been recently updated (12). Exclusive BR measurements need to be compared with unclean theoretical expectations, due to the presence of large long distance contributions (13). Anyway, it is possible to measure some interesting 


\begin{tabular}{lccc}
\hline Decay Mode & Combined & $0.1<m_{l l}^{2}<7.02 \mathrm{GeV}^{2} / \mathrm{c}^{4}$ & $m_{l l}^{2}>10.24 \mathrm{GeV}^{2} / \mathrm{c}^{4}$ \\
\hline$K^{+} \ell^{+} \ell^{-}$ & $-0.18_{-0.18}^{+0.18} \pm 0.01$ & $-0.18_{-0.19}^{+0.19} \pm 0.01$ & $-0.09_{-0.39}^{+0.36} \pm 0.02$ \\
$K^{* 0} \ell^{+} \ell^{-}$ & $0.02_{-0.20}^{+0.20} \pm 0.02$ & $-0.23_{-0.38}^{+0.38} \pm 0.02$ & $0.17_{-0.24}^{+0.24} \pm 0.02$ \\
$K^{*+} \ell^{+} \ell^{-}$ & $0.01_{-0.24}^{+0.26} \pm 0.02$ & $0.10_{-0.24}^{+0.25} \pm 0.02$ & $-0.18_{-0.55}^{+0.45} \pm 0.04$ \\
$K^{*} \ell^{+} \ell^{-}$ & $0.01_{-0.15}^{+0.16} \pm 0.01$ & $0.01_{-0.20}^{+0.21} \pm 0.01$ & $0.09_{-0.21}^{+0.21} \pm 0.02$ \\
\hline
\end{tabular}

Table 1: Forward backward asymmetry for two different $m_{l l}^{2}$ region and in the full range.

rate asymmetry that are sensitive to NP effects (14), with part of the theoretical uncertainties canceling when taking the ratios. The most important one is the forward-backward asymmetry. A preliminary result by the $B A B A R$ Collaboration, from a sample of about $384 \times 10^{6} B \bar{B}$ pairs, is quoted in Tab. 1 for two different ranges of the dilepton invariant mass $m_{l l}^{2}$.

Concerning the $B \rightarrow K^{(*)} \nu \bar{\nu}$ BRs, the Collaboration recently presented an update of the search for the $K^{+}$mode and the first preliminary BABAR search for the $K^{* \pm, 0}$ modes. They are performed by means of a recoil technique, using semileptonic $B \rightarrow D^{(*)} \ell \nu$ decays for the $B_{\text {tag }}$ reconstruction. Then a $K^{(*)}$ is looked for in the recoil, requiring that no extra track is present.

Since NP effects can strongly affect the kinematics of the $B \rightarrow K^{*} \nu \bar{\nu}$ decay, for the first time this search has been performed by using only the variables that are not correlated to the assumed kinematical model.

In the $B^{+} \rightarrow K^{+} \nu \bar{\nu}$ analysis, the signal yield is extracted with a counting technique, after a multivariate selection based on the random forest algorithm (15). In the $B \rightarrow K^{*} \nu \bar{\nu}$ search the yield is extracted by means of a fit to the distribution of $E_{\text {extra }}$, defined as the sum of the energies of the neutral particles not associated either to the $B_{\text {tag }}$ or the signal $K^{(*)}$.

The following upper limits at $90 \%$ confidence level (CL) have been set:

$$
\begin{aligned}
\mathcal{B}\left(B^{+} \rightarrow K^{+} \nu \bar{\nu}\right) & <4.2 \times 10^{-5} \\
\mathcal{B}\left(B^{0} \rightarrow K^{* 0} \nu \bar{\nu}\right) & <18 \times 10^{-5} \\
\mathcal{B}\left(B^{+} \rightarrow K^{*+} \nu \bar{\nu}\right) & <9 \times 10^{-5}
\end{aligned}
$$

based on about $351 \times 10^{6}\left(\right.$ for $\left.K^{+} \nu \bar{\nu}\right)$ and $454 \times 10^{6}$ (for $\left.K^{*} \nu \bar{\nu}\right) B \bar{B}$ pairs. These ULs are about a factor 10 above the expected SM values.

\section{Leptonic $B$ decays}

Purely leptonic $B$ decays occur in the SM through annihilation diagrams, and hence are highly suppressed. According to the SM, the only among them that is accessible at the present $B$ Factories is the $B^{+} \rightarrow \tau^{+} \nu$ decay, whose BR is expected to be at the level of $10^{-4}$. On the other hand, enhancements are possible in some NP scenarios.

The most recent BABAR measurements for $B^{+} \rightarrow \tau^{+} \nu$ have been performed in the recoil of both hadronic and semileptonic $B_{\text {tag }}$ decays (16). The $\tau$ decay modes used in these analyses are both leptonic and hadronic. The measured BR is:

$$
\begin{aligned}
\mathcal{B}\left(B^{+} \rightarrow \tau^{+} \nu\right) & =(0.9 \pm 0.6 \pm 0.1) \\
\mathcal{B}\left(B^{+} \rightarrow \tau^{+} \nu\right) & =\left(1.8_{-0.8}^{+0.9} \pm 0.4\right)
\end{aligned}
$$

for the semileptonic and hadronic analysis respectively, based on $383 \times 10^{6} B \bar{B}$ pairs. They are in agreement with the expected SM values. 
The BABAR Collaboration also performed searches for the $B \rightarrow \ell^{+} \ell^{-}$decays, where the $e^{+} e^{-}$, $\mu^{+} \mu^{-}, e^{+} \mu^{-}, e^{+} \tau^{-}$and $\mu^{+} \tau^{-}$combinations are considered. If there is no $\tau$ in the final, state, a full reconstruction is performed and the signal yield is extracted with a ML fit to the distributions of $m_{E S}$ and $\Delta E$. If a $\tau$ is present, a hadronic recoil technique is adopted and the momentum of the other lepton is fitted in order to extract the signal yield. The following ULs at 90\% CL are set:

$$
\begin{gathered}
\mathcal{B}\left(B^{0} \rightarrow e^{+} e^{-}\right)<11.3 \times 10^{-8} \\
\mathcal{B}\left(B^{0} \rightarrow \mu^{+} \mu^{-}\right)<5.2 \times 10^{-8} \\
\mathcal{B}\left(B^{0} \rightarrow e^{+} \mu^{-}\right)<9.2 \times 10^{-8} \\
\mathcal{B}\left(B^{0} \rightarrow e^{+} \tau^{-}\right)<2.8 \times 10^{-5} \\
\mathcal{B}\left(B^{0} \rightarrow \mu^{+} \tau^{-}\right)<2.2 \times 10^{-5}
\end{gathered}
$$

\section{LFV $\tau$ decays}

The decays of the $\tau$ lepton can be studied at BABAR thanks to the high $e^{+} e^{-} \rightarrow \tau^{+} \tau^{-}$cross section at $10.58 \mathrm{GeV}(\sim 0.92 \mathrm{nb})$. The most recent measurements published by the Collaboration for the LFV $\tau$ decays concern $\tau^{+} \rightarrow \ell^{+} \omega$ (17) and $\tau^{+} \rightarrow \ell^{+} \ell^{-} \ell^{+}$(18). These decays are expected to be unaccessible in the SM hypothesis but they can be enhanced and reach the $10^{7}$ level in some supersymmetric scenario.

These analyses are performed by looking for a 1-3 topology: the event is divided in two hemispheres and only one track (assumed to come from a leptonic $\tau$ decay) is searched for in one hemisphere, while three tracks (the signal signature) are searched for in the opposite hemisphere. Then, two kinematical variables, the missing energy $\Delta E$ and the beam energy constrained $\tau$ mass $m_{E C}$, are used to define a signal region. Finally, the observed events in the signal region are compared to the expected yields extracted from MC simulations. The following ULs are set:

$$
\begin{aligned}
\mathcal{B}\left(\tau^{+} \rightarrow e^{+} \omega\right) & <10 \times 10^{-8} \\
\mathcal{B}\left(\tau^{+} \rightarrow \mu^{+} \omega\right) & <11 \times 10^{-8} \\
\mathcal{B}\left(\tau^{+} \rightarrow e^{+} e^{-} e^{+}\right) & <10 \times 10^{-8} \\
\mathcal{B}\left(\tau^{+} \rightarrow \mu^{+} e^{-} e^{+}\right) & <11 \times 10^{-8} \\
\mathcal{B}\left(\tau^{+} \rightarrow \mu^{-} e^{+} e^{+}\right) & <10 \times 10^{-8} \\
\mathcal{B}\left(\tau^{+} \rightarrow e^{-} \mu^{+} \mu^{+}\right) & <11 \times 10^{-8} \\
\mathcal{B}\left(\tau^{+} \rightarrow e^{+} \mu^{-} \mu^{+}\right) & <10 \times 10^{-8} \\
\mathcal{B}\left(\tau^{+} \rightarrow \mu^{+} \mu^{-} \mu^{+}\right) & <11 \times 10^{-8}
\end{aligned}
$$

\section{References}

[1] B. Aubert et al. [BABAR Collaboration], Nucl. Instrum. Meth. A 479 (2002) 1

[2] S. Agostinelli et al. [GEANT4 Collaboration], Nucl. Instrum. Meth. A 506 (2003) 250.

[3] M. Misiak et al., Phys. Rev. Lett. 98 (2007) 022002

[4] T. Hurth, E. Lunghi and W. Porod, Nucl. Phys. B 704 (2005) 56 
[5] B. Aubert et al. [BABAR Collaboration], Phys. Rev. D 72 (2005) 052004

[6] B. Aubert et al. [BABAR Collaboration], Phys. Rev. D 77 (2008) 051103

[7] G. Buchalla, G. Hiller and G. Isidori, Phys. Rev. D 63 (2001) 014015.

[8] C. Bird, P. Jackson, R. Kowalewski and M. Pospelov, Phys. Rev. Lett. 93 (2004) 201803.

[9] T. M. Aliev, A. S. Cornell and N. Gaur, JHEP 0707 (2007) 072

[10] B. Aubert et al. [BABAR Collaboration], Phys. Rev. Lett. 93 (2004) 081802

[11] B. Aubert et al. [BABAR Collaboration], arXiv:0805.4796 [hep-ex].

[12] B. Aubert et al. [BABAR Collaboration], arXiv:0807.4119 [hep-ex].

[13] M. Beneke, T. Feldmann and D. Seidel, Nucl. Phys. B 612 (2001) 25

[14] T. Feldmann and J. Matias, JHEP 0301 (2003) 074 C. Bobeth, G. Hiller and G. Piranishvili, JHEP 0807 (2008) 106

[15] L. Breiman, Machine Learning 45, 5-32 (2001).

[16] B. Aubert et al. [BABAR Collaboration], Phys. Rev. D 76 (2007) 052002 B. Aubert et al. [BABAR Collaboration], Phys. Rev. D 77 (2008) 011107

[17] B. Aubert et al. [BABAR Collaboration], Phys. Rev. Lett. 100 (2008) 071802

[18] B. Aubert et al. [BABAR Collaboration], Phys. Rev. Lett. 99 (2007) 251803 